\title{
我
}

\section{Electrical Characteristics of Poly(ethylene oxide)-urea Complex Films}

\author{
Mi Yeon Cho, Kyoungah Cho, and Sangsig Kim ${ }^{+}$ \\ Department of Electrical Engineering, Korea University, Seoul 136-713, Korea
}

Received January 3, 2012; Revised February 23, 2012; Accepted March 6, 2012

\begin{abstract}
The electrical characteristics of complex films composed of poly(ethylene oxide) (PEO) and urea as a function of the urea concentration were examined in this study. Moreover, their structural characteristics were also compared. Depending on the urea concentration, the structural phases were classified as PEO+ $\beta$-phase composite, $\beta$-phase $+\alpha$ phase composites, or $\alpha$-phase composite+urea. At urea concentrations below $\sim 0.064 \mathrm{M}$, the $\beta$-phase was dominant in the complex film. Moreover, the conductance increased rapidly with an increase in the urea concentration. For urea concentrations ranging from $\sim 0.064$ to $\sim 0.25 \mathrm{M}$, the $\beta$-phase was gradually substituted by the $\alpha$-phase. As the film was composed entirely of the $\alpha$-phase at urea concentrations greater than $\sim 0.25 \mathrm{M}$, its conductance was decreased. In this study, the electrical characteristics observed for the different phases are analyzed and discussed.
\end{abstract}

Keywords: PEO-urea complex, Conductivity, Phase-transition, Composite film

\section{INTRODUCTION}

Inclusion Compounds (ICs) including PEO-urea complexes have extraordinary structures and each polymer chain is surrounded by nano-channels that are composed of small molecules. The structures of ICs have been studied with much interest, as the characteristics of each polymer chain exclude the interactions with other polymer chains and this differs from the characteristics of bundles of other ordinary polymers [1]. Studies of PEO-urea complexes have focused particularly on the extent of the unique structures of ICs [2-6]. PEO-urea complexes are known to form two different structural phases. One is the stable $\alpha$-phase complexes with a urea/PEO ratio of 1.8 and the other is the metastable $\beta$-phase complexes with a urea/PEO ratio of 1.0. Some parts of the $\beta$-phase complexes can be transformed into $\alpha$-phase complexes by increasing the urea mole fraction or by heating. The $\beta-\alpha$ transition of PEO-urea complexes and their structural phase diagrams have been intensively studied [2,5-10].

${ }^{\dagger}$ Author to whom all correspondence should be addressed: E-mail: sangsig@korea.ac.kr

Copyright $@ 2012$ KIEEME. All rights reserved.

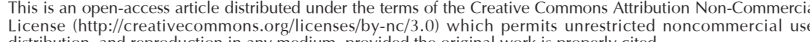
distribution, and reproduction in any medium, provided the original work is properly cited.
While their fundamental chemical properties, structural phases, and stabilities have been widely reported, very little is known about the electrical I-V characteristics or conductivities of PEOurea complexes. In this study, the dependence of the electrical characteristics on the phase of PEO-urea complex films was investigated. The three different phases of the complex films were first examined and then, the electrical I-V characteristics obtained from the different phase composites were compared with the corresponding phases. Finally, the dependence of the electrical characteristics on the phase of complex films was analyzed and discussed.

\section{EXPERIMENTS}

Poly(ethylene oxide) (PEO, $\mathrm{Mw}=900,000 \mathrm{~g} / \mathrm{mol}$ ) and urea (Sigma-Aldrich, U5378) were used without further purification. The chemical structures of the PEO and urea molecules are shown in the inset of Fig. 1. $0.1 \mathrm{~g}$ of $\mathrm{PEO}$ powder was mixed with various concentrations of urea $(0 \sim 1 \mathrm{M})$ in $1 \mathrm{ml}$ of D.I. water and then stirred for $2 \mathrm{~h}$. For the FT-IR, XRD, and SEM measurements, the mixture solutions of PEO and urea were spin-coated on glass substrates at $800 \mathrm{rpm}$ for $30 \mathrm{~s}, 2,000 \mathrm{rpm}$ for $90 \mathrm{~s}$, and 5,000 rpm for $30 \mathrm{~s}$, sequentially. In order to examine their electrical 


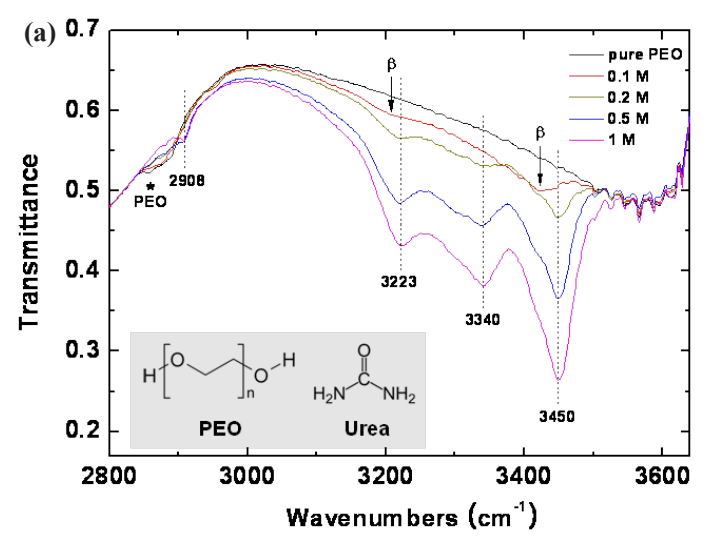

(b)

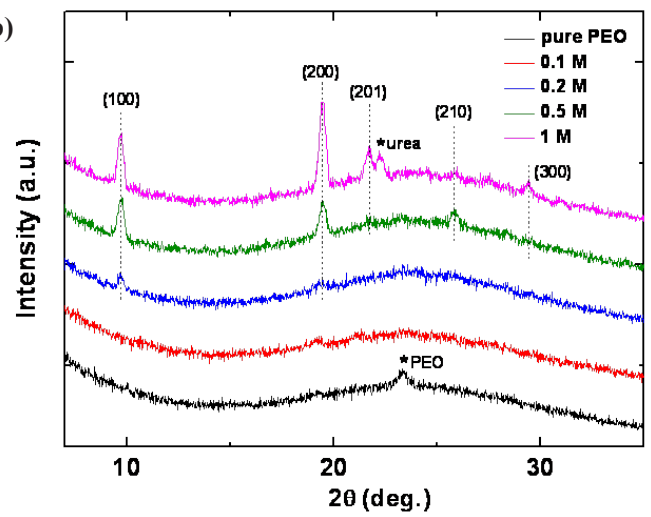

Fig. 1. (a) FT-IR spectra and (b) XRD patterns of the PEO-urea complex films with various concentrations of urea. The dots and arrows denote the $\alpha$ - and $\beta$-phases of the PEO-urea complex, respectively, and the asterisks $\left(^{*}\right)$ denote pure PEO or pure urea.

characteristics, the PEO-urea complex films were formed on patterned glass substrates with gold electrodes that were thermally deposited by using a finger pattern metal mask with a length of $500 \mu \mathrm{m}$. The dc conductivities were obtained through four-probe measurements. The dimensions of the complex film present between the two adjacent electrodes of the four Au electrodes were about a length of $100 \mu \mathrm{m}$ and width of $3 \mathrm{~mm}$. All the measurements were taken at room temperature.

\section{RESULTS AND DISCUSSION}

Figure 1(a) shows the FT-IR spectra of the PEO-urea complex films with various concentrations of urea $(0 \sim 1 \mathrm{M})$. First, the spectrum of the pure PEO film is characterized by examining the symmetric stretching vibrations of the $\mathrm{CH}_{2}$ groups of the PEO chains that were present at 2,857 2,888 $\mathrm{cm}^{-1}$. The PEO-urea complex bands are identified by the $\mathrm{CH}_{2}$ groups at $2,908 \mathrm{~cm}^{-1}$ and the N-H stretching bands near 3,223, 3,340 and 3,450 $\mathrm{cm}^{-1}$. The $\mathrm{N}-\mathrm{H}$ stretching bands are related to the hydrogen bonds in the molecular complex that was formed between the ether oxygen of PEO and the $\mathrm{NH}_{2}$ groups of urea [3], so that they appear in the $\alpha$-phase or $\beta$-phase depending on the stoichiometric relationship (more specifically, urea/PEO $=1.8$ for the $\alpha$-phase and 1.0 for the $\beta$-phase).

As shown in Fig. 1(a), the N-H stretching bands in the spectrum of the film with a urea concentration of $0.1 \mathrm{M}$ were observed at lower frequencies compared to those in the spectra of the film with urea concentrations of more than $0.2 \mathrm{M}$. This indicates that the complex with a urea concentration of $0.1 \mathrm{M}$
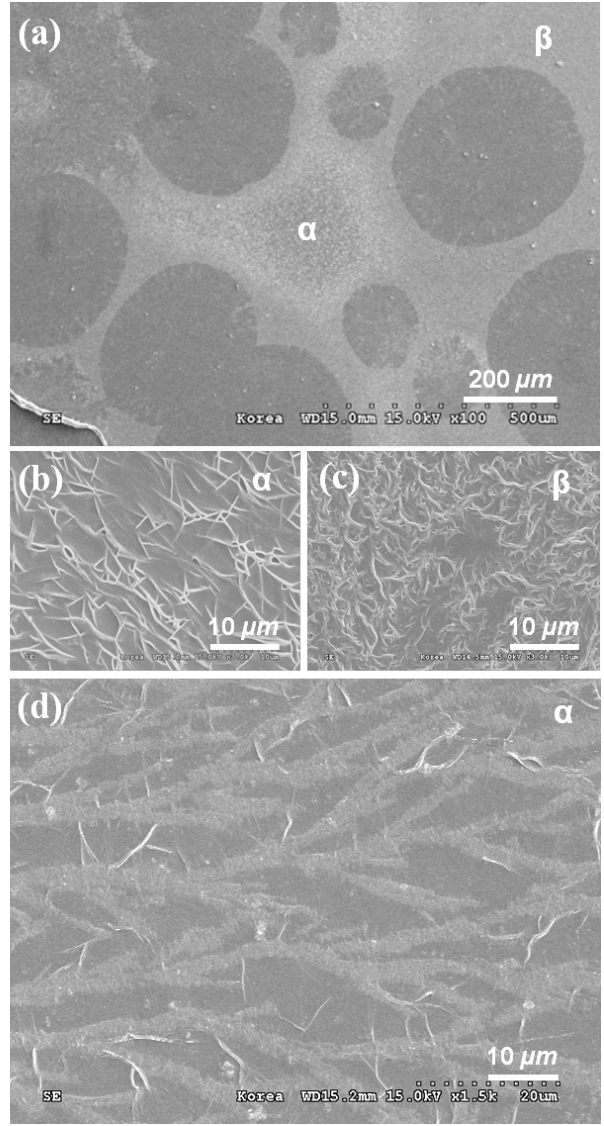

Fig. 2. SEM images of the PEO-urea complex films for urea concentrations of (a)-(c) $0.2 \mathrm{M}$ and (d) $0.3 \mathrm{M}$. (b) and (c) are the enlarged SEM images of the $\alpha$ - and $\beta$-phase regions of (a).

consists mainly of the $\beta$-phase and that the other complexes with urea concentrations of more than $0.2 \mathrm{M}$ consist mainly of the $\alpha$-phase [9]. Therefore, the $\beta$-phase of the complex transforms into the $\alpha$-phase with an increase in the urea concentration [4]: this transformation is hereafter, referred to as the $\beta$ - $\alpha$ transition. Figure 1(b) shows the XRD patterns of the complex films with various concentrations of urea $(0 \sim 1 \mathrm{M})$. The pure PEO film exhibited the PEO characteristic peak that appears at a $2 \theta$ angle of $23.2^{\circ}$. For urea concentrations higher than $0.2 \mathrm{M}$, the $\alpha$-phase characteristic peaks of the complex films were assigned to the $100,200,201,210$ and 300 planes of the $\alpha$-phase [6], as shown in Fig. 1(b). Moreover, at a urea concentration of $1 \mathrm{M}$, a pure urea peak was also observed at $22.2^{\circ}$. Note that, for comparison, the distinct XRD peaks of the $\alpha$-phase obtained from relatively high urea concentrations of 0.5 and $1 \mathrm{M}$ are also added in Fig. 1(b). However, definite peaks of the $\beta$-phase were not observed in this $\mathrm{XRD}$ study, perhaps this is due to the relatively low crystallinity of the $\beta$-phase.

The SEM images of the PEO-urea complex films are exhibited in Fig. 2. In Fig. 2(a), both the $\alpha$ - and $\beta$-phases are present in a single complex film with a urea concentration of $0.2 \mathrm{M}$. The negative spherulites that are seen in Fig. 2(a) correspond to the PEO chains. The existence of the spherulites implies that some of the PEO chains escaped from the $\beta$-phase complex (urea/PEO=1.0) during the $\beta-\alpha$ transition [4]. Note that a higher urea ratio is required for the $\alpha$-phase complex (urea/PEO=1.8). The enlarged SEM images of the $\alpha$ - and $\beta$-phase parts shown in Fig. 2(a) are presented in Figs. 2(b) and (c), respectively. A careful comparison of the SEM images reveals that the shape of the $\alpha$-phase 

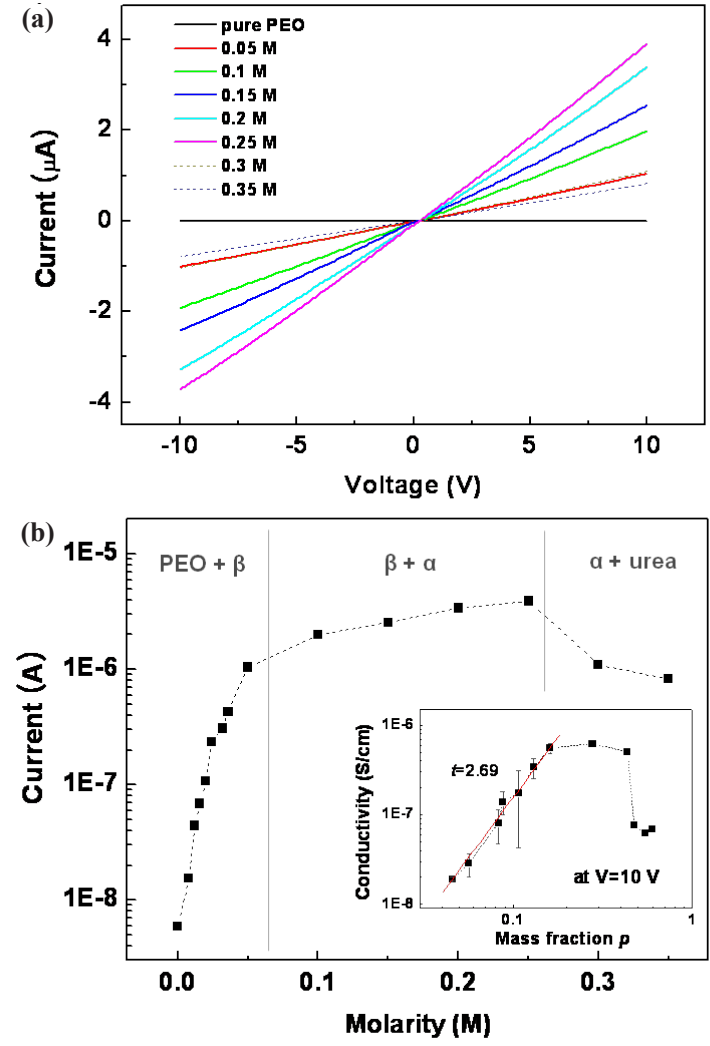

Fig. 3. (a) $I-V$ characteristics and (b) $\log -\log$ plot of $I$ as a function of concentration of urea for the PEO-urea complex films. Inset: log-log plot of dc conductivity as a function of mass fraction $(p)$.

consists of straight tree-shaped fractals, while the $\beta$-phase has the shape of curly random-networks. At urea concentrations that were higher than $\sim 0.25 \mathrm{M}$, the $\beta$-phase is absent and the $\alpha$-phase with tree-shaped fractals having thicker branches covers the glass substrate entirely. For relatively higher concentrations, the $\beta$-phase transforms into the $\alpha$-phase, and all the bundles of the PEO chains with the shape of negative spherulites are used up to form the $\alpha$-phase. Figure 2(d) demonstrates that the film with a urea concentration of $0.3 \mathrm{M}$ consists only of the $\alpha$-phase.

The current-voltage $(I-V)$ characteristics of the PEO-urea complex films with various concentrations of urea are shown in Fig. 3(a). As the urea concentration varies from 0 to $\sim 0.25 \mathrm{M}$, the current increases in magnitude. On the other hand, for urea concentrations of $0.3 \mathrm{M}$ and $0.35 \mathrm{M}$, the current drops rapidly in magnitude. This takes place after the occurrence of the $\beta-\alpha$ phase transition. The change in the magnitude of the current at a bias voltage of $10 \mathrm{~V}$ as a function of the urea concentration is summarized in a logarithmic scale in Fig. 3(b). The characteristics of the changes in the magnitude of the current can be classified as three different characteristics that correspond to the PEO+ $\beta$ phase, $\beta-+\alpha$-phases, and $\alpha$-phase+urea. For urea concentrations below $\sim 0.06 \mathrm{M}$, the log-scaled magnitude of the current increases rapidly and this indicates the presence of the $\mathrm{PEO}+\beta$-phase. In the $\beta-+\alpha$-phases of the complex films with urea concentrations ranging from $\sim 0.06$ to $\sim 0.25 \mathrm{M}$, the log-scaled magnitude of the current increases gradually. For urea concentrations that are higher than $\sim 0.25 \mathrm{M}$, the decrease in the magnitude of the current indicates the presence of the $\alpha$-phase+urea. The inset of
Fig. 3(b) shows the dc conductivity $\left(\sigma_{\mathrm{dc}}\right)$ of the complex films as a function of the mass fraction $(p)$ of urea in a logarithmic scale. The $\sigma_{\mathrm{dc}}(p)$ of the PEO-urea complex films increased logarithmically and then, it was saturated as the mass fraction of urea increased logarithmically. These characteristics are reflected by the percolation behaviors and $\sigma_{\mathrm{dc}}(p)$ follows the scaling law of the percolation theory. It is briefly expressed as $\sigma_{\mathrm{dc}}(p) \infty\left|p-p_{\mathrm{c}}\right|^{\mathrm{t}}[11]$. The slope of the plot in the inset that corresponds to the critical exponent $t$, changes from 2.69 to $\sim 0$ and this implies a percolation threshold at $p \sim 0.16$ (at a urea concentration of $\sim 0.064 \mathrm{M}$ ). The percolation threshold indicates the beginning of the - transition of the PEO-urea complex.

\section{SUMMARY}

The structural and electrical characteristics of the PEO-urea complex films prepared in this study were investigated using various molar ratios. As the urea concentration increases, the complex films exhibit a metastable $\beta$-phase and stable $\alpha$-phase sequentially. The conductance of the complex films increases rapidly and then, gradually with an increasing urea concentration. These electrical characteristics can be explained by the percolation behaviors of the films with the percolation threshold at $p \sim 0.16$ (at $\sim 0.064 \mathrm{M}$ ). The percolation threshold indicates the beginning of the $\beta-\alpha$ transition.

\section{ACKNOWLEDGMENTS}

This work was partly supported by the Nano R\&D Program (M10703000980-08M0300-98010), Seoul R\&D Program (PA090914), and the World Class University (WCU, R32-2008000-10082-0) Project of the Ministry of Education, Science and Technology (Korea Science and Engineering Foundation).

\section{REFERENCES}

[1] J. Lu, P. A. Mirau, and A. E. Tonelli, Prog. Polym. Sci. 27, 357 (2002) [DOI: 10.1016/S0079-6700(01)00045-4].

[2] H. Tadokoro, T. Yoshihara, Y. Chatani, and S. Murahashi, Polym. Lett. 2, 363 (1964) [DOI: 10.1002/pol.1964.110020410].

[3] B. G. Bogdanov, M. Michailov, C. V. Uzov, and G. G. Cavrailova, J. Polym. Sci. 32, 387 (1994) [DOI: 10.1002/polb.1994.090320222].

[4] H. Ye, M. Peng, J. Xu, B. Guo, Q. Chen, T. Yun, and H. Ma, Polymer 48, 7364 (2007) [DOI: 10.1016/j.polymer.2007.10.033].

[5] A. Chenite and F. Brisse, Macromol. 24, 2221 (1991) [DOI: 10.1021/ma00009a015].

[6] J. Wagner, M. Dosiere, and J. Guenet, Macromol. Symp. 222, 121 (2005) [DOI: 10.1002/masy.200550414]

[7] K. Suehiro and Y. Nagano, Makromol. Chem. 184, 669 (1983) [DOI: 10.1002/macp.1983.021840319].

[8] Y. Liu and C. Pellerin, Macromol. 39, 8886 (2006) [DOI: 10.1021/ ma0625408].

[9] Y. Liu, H. Antaya, and C. Pellerin, J. Polym. Sci. B Polym. Phys. 46, 1903 (2008) [DOI: 10.1002/polb.21523].

[10] Y. Liu and C. Pellerin, Polymer 50, 2601 (2009) [DOI: 10.1016/ j.polymer.2009.03.050].

[11] H. M. Kim, K. Kim, C. Y. Lee, J. Joo, S. J. Cho, H. S. Yoon, D. A. Pejakovic', J.W. Yoo, and A.J. Epstein, Appl. Phys. Lett. 84, 589 (2004) [DOI: 10.1063/1.1641167]. 